\title{
THE
}

\section{Tuning a Polar Molecule for Selective Cytoplasmic Delivery by a pH (Low) Insertion Peptide}

Dayanjali Wijesinghe

University of Rhode Island

Donald M. Engelman

Oleg A. Andreev

University of Rhode Island, andreev@uri.edu

Yana K. Reshetnyak

University of Rhode Island, reshetnyak@uri.edu

Follow this and additional works at: https://digitalcommons.uri.edu/phys_facpubs

This is a pre-publication author manuscript of the final, published article.

Terms of Use

All rights reserved under copyright.

\section{Citation/Publisher Attribution}

Wijesinghe, D., Engelman, D. M., Andreev, O. A., \& Reshetnyak, Y. K. (2011). Tuning a Polar Molecule for Selective Cytoplasmic Delivery by a pH (Low) Insertion Peptide. Biochemistry, 50(47), 10215-10222. doi: 10.1021/bi2009773

Available at: https://doi.org/10.1021/bi2009773

This Article is brought to you for free and open access by the Physics at DigitalCommons@URI. It has been accepted for inclusion in Physics Faculty Publications by an authorized administrator of DigitalCommons@URI. For more information, please contact digitalcommons-group@uri.edu. 


\title{
Tuning a polar molecule for selective cytoplasmic delivery by a pH (Low) Insertion Peptide
}

\author{
Dayanjali Wijesinghe ${ }^{1}$, Donald M. Engelman ${ }^{2}$, Oleg A. Andreev ${ }^{1}$, and Yana K. Reshetnyak ${ }^{1}$ \\ ${ }_{1}^{1}$ Physics Department, University of Rhode Island, 2 Lippitt Rd., Kingston, RI, 02881, USA \\ ${ }^{2}$ Department of Molecular Biophysics and Biochemistry, Yale University, P.O. Box 208114, New \\ Haven, CT 06520, USA
}

\begin{abstract}
Drug molecules are typically hydrophobic and small in order to traverse membranes to reach cytoplasmic targets, but we have discovered that more polar molecules can be delivered across using water-soluble, moderately hydrophobic membrane peptides of the $\mathrm{pHLIP}^{\circledR}$ (pH Low Insertion Peptide) family. Delivery of polar cargo molecules could expand the chemical landscape for pharmacological agents that have useful activity but are too polar by normal drug criteria. The spontaneous insertion and folding of the pHLIP peptide across a lipid bilayer seeks a free energy minimum, and insertion is accompanied by a release of energy that can be used to translocate cellimpermeable cargo molecules. In this study we report our first attempt to tune the hydrophobicity of a polar cargo, phallacidin, in a systematic manner. We present the design, synthesis and characterization of three phallacidin cargoes, where the hydrophobicity of the cargo was tuned by the attachment of diamines of various lengths of hydrophobic chains. The phallacidin cargoes were conjugated to pHLIP, and shown to selectively inhibit the proliferation of cancer cells in a concentration-dependent manner at low $\mathrm{pH}$.
\end{abstract}

\section{Keywords}

pHLIP; phallacidin; hydrophobicity; translocation across membrane; drug design; drug delivery; acidity; solubility

\section{Introduction}

Targeted drug delivery would allow drugs to preferentially affect diseased cells, enhancing therapeutic efficacy while reducing side effects. Targeting could be particularly important for cancer therapy, since most anti-cancer drugs are toxic, not only killing cancer cells but also causing serious damage to healthy tissues. Despite significant progress toward drugs that specifically target protein biomarkers for certain kinds of cancer cells, there is still no "silver bullet" against cancer. One reason for the limited success so far is that cells in tumors are heterogeneous and selection for resistance to protein-targeted drugs and to the immune system can occur (1). An alternative might be to find a targeting mechanism that does not depend on a selectable marker. One of the universal differences between cancerous and normal tissues is that the former exhibit a significantly acidic extracellular environment. Acidosis is a hallmark of solid tumor development at both very early and advanced stages,

Corresponding Authors: Yana K. Reshetnyak, Physics Department, University of Rhode Island, 2 Lippitt Rd., Kingston, RI, 02881, USA, Phone: (401)-874-2060, Fax: (401) 874-2380, reshetnyak@mail.uri.edu. 
as a consequence of anaerobic metabolism (the Pasteur effect) (2) and "aerobic glycolysis" known as the Warburg effect (3).

To target acidic tissues, we have developed a new approach, based on the action of a watersoluble, moderately hydrophobic membrane polypeptide, pHLIP (pH Low Insertion Peptide), derived from the bacteriorhodopsin C helix. pHLIP is a soluble peptide that was found to fold and insert across a membrane to form a stable transmembrane alpha helix under acidic conditions (4). When triggered by low $\mathrm{pH}$, members of the pHLIP peptide family act as monomeric, membrane-inserting peptides that translocate their carboxyl termini across membranes into the cytoplasms of targeted cells, while the amino termini remain in the extracellular space, locating the peptide across the plasma membrane lipid bilayers (5). Because the insertion occurs at low $\mathrm{pH}$, acidic tissues are targeted, and so pHLIP has a dual delivery capability: it can tether N-terminus linked cargo molecules to the surfaces of cells in diseased tissues and it can move a C-terminus linked, cell-impermeable cargo molecule across the membrane into the cytoplasm using the free energy of insertion and folding $(6,7)$. Use of a cleavable link, such as a disulfide, releases the cargo inside the cell $(8,9)$. Low $\mathrm{pH}$ leads to the protonation of negatively charged residues (Asp or Glu), which enhances peptide hydrophobicity, increasing the affinity of the peptide for the lipid bilayer and triggering peptide folding and subsequent membrane insertion (10-12). The source of energy for moving polar molecules attached to pHLIP through the hydrophobic layer of a membrane is the membrane-associated folding of the polypeptide (13-15). The affinity of peptide for a membrane at low $\mathrm{pH}$ is about 5 times higher than at high $\mathrm{pH}$, allowing pHLIP to distinguish and mark acidic diseased tissue $(10,16,17)$ associated with various pathological states, such as cancerous tumors, inflammation, ischemia, stroke and others.

Our recent findings indicate that pHLIP facilitates the translocation of phalloidin, a cellimpermeable polar toxin, which leads to the inhibition of the proliferation of cancer cells in a pH-dependent fashion (8). However, the antiproliferative effect was observed only when a hydrophobic facilitator (rhodamine) was attached to the peptide inserting end. An alternative approach is to modify the properties of the cargo molecule to optimize delivery, and the goal of this study is to tune properties of the pHLIP-cargo constructs to achieve the most efficient $\mathrm{pH}$-dependent translocation of cargo molecules across the lipid bilayer. The properties of a molecule delivered into cells will help to define the chemical landscape available for the use of pHLIP. Here we present the design, synthesis and characterization of three phallacidin cargoes, where the hydrophobicity of the cargo was tuned by attachment of diamines having various lengths of hydrophobic chains. Three constructs, where pHLIP was linked to phallcidin cargoes of different polarity were synthesized, characterized, and tested the antiproliferative effect on cultured cells.

\section{Materials and Methods}

\section{Peptide preparation}

The pHLIP peptide (AEQNPIYWARYADWLFTTPLLLLDLALLVDADEGCT) was prepared by solid-phase peptide synthesis at the W.M. Keck Foundation Biotechnology Resource Laboratory at Yale University. The lyophilized powder was soluble in $3 \mathrm{M}$ urea or DMSO (dimethyl sulfoxide). When dissolved in urea the peptide was transferred to buffer using a G-10 size-exclusion spin column. The concentration of the peptide was determined spectrophotometricly by measuring absorbance at $280 \mathrm{~nm}\left(\varepsilon_{280}=13,940 \mathrm{M}^{-1} \mathrm{~cm}^{-1}\right)$. 


\section{Synthesis of phallacidin- $\left(\mathrm{CH}_{2}\right)_{n}-\mathrm{SH}$}

Materials-Phallacidin was purchased from GenoLite Biotek, $N$-hydroxysuccinimide (NHS), $N, N$ '-dicyclohexylcarbodiimide (DCC), $N$-succinimidyl 3-(2-pyridyl-dithio)propionate (SPDP) were from Thermo Scientific, Hexamethylenediamine 98\%, 1,4diaminobutane 99\%, 1,10-diaminodecane 97\% were from Sigma Aldrich.

Step1-Phallacidin $(2.6 \mathrm{mg}, 3.10 \mu \mathrm{mol})$ was dissolved in $100 \mu \mathrm{l}$ dry DMF (dimethylformamide) and transferred into a $1.5 \mathrm{ml}$ glass vial followed by addition of NHS ( $2.5 \mathrm{mg}, 21.6 \mu \mathrm{mol}, 7 \mathrm{eq})$ in $30 \mu \mathrm{l}$ dry DMF and mixed well. DCC (1.15 mg, $5.57 \mu \mathrm{mol}, 1.8$ eq) was added to the reaction mixture. The reaction mixture was stirred at room temperature overnight, then the reaction mixture was centrifuged and separated from the urea crystals. The progress of the reaction was monitored by reverse phase HPLC (high-performance liquid chromatography) at $\mathrm{t}=0$ and at $\mathrm{t}=12 \mathrm{hrs}$. (Agilent Technologies Zorbax SB-C184.6 $\times$ $250 \mathrm{~mm}$ column; flow rate $1 \mathrm{ml} / \mathrm{min}$; phase A water $+0.05 \%$ TFA (trifluoroacetic acid); phase $\mathrm{B}$ acetonitrile $+0.05 \% \mathrm{TFA}$; gradient $30 \mathrm{~min}$ from 95:5 A/B to 50:50 A/B) The phallacidin starting material elutes at $24.9 \mathrm{~min}$, while activated hydroxysuccinimidephallacidin elutes at $23.6 \mathrm{~min}$. The reaction was completed by $12 \mathrm{hrs}$.

Step2-The supernatant from the step 1, which contained the activated hydroxysuccinimidephallacidin was added into diamines $\mathrm{H}_{2} \mathrm{~N}-\left(\mathrm{CH}_{2}\right)_{\mathrm{n}}-\mathrm{NH}_{2}(\mathrm{n}=4,6$ or 10) dissolved in dry DMF ( $31 \mu \mathrm{mol}, 10$ eq of phallacidin) and the reaction mixture was stirred at room temperature for $10 \mathrm{hrs}$. The addition of the diamine resulted in the formation of a precipitate: dehydrated phallacidin diamine salt. The product, phallacidin- $\left(\mathrm{CH}_{2}\right)_{n} \mathrm{NH}_{2}$ (phallCn) was found both in the precipitate and in the supernatant. The precipitate was separated by centrifugation and dissolved in $120 \mu \mathrm{MeOH} / \mathrm{H}_{2} \mathrm{O}(1: 1)$. The product was purified using HPLC and lyophilized. The elution times of phallacidin- $\left(\mathrm{CH}_{2}\right)_{4} \mathrm{NH}_{2}$ (phallC4), phallacidin- $\left(\mathrm{CH}_{2}\right)_{6} \mathrm{NH}_{2}$ (phallC6) and phallacidin- $\left(\mathrm{CH}_{2}\right)_{10} \mathrm{NH}_{2}$ (phallC10) were $23.1,24.6$ and $29.5 \mathrm{~min}$, respectively as expected from the increasing hydrophobicities. The lyophilized powder was then dissolved in $100 \mu \mathrm{l}$ of $\mathrm{MeOH} / \mathrm{H}_{2} \mathrm{O}(1: 1)$, quantified by measuring OD (optical density) at $300 \mathrm{~nm}\left(\varepsilon_{300}\right.$ of phallacidin: $\left.10,100 \mathrm{M}^{-1} \mathrm{~cm}^{-1}\right)$ and analyzed using ESI (electronspray ionization) mass spectrometry. Molecular weights (MW) of the phallotoxins phallC4, phallC6 and phallC10 were 917.23, 945.18 and 1001.22 Da, respectively (the expected MWs are 917.06, 945.12 and 1001.23 Da).

Step 3-The products from step 2 (in $100 \mu \mathrm{l}$ of $\mathrm{MeOH} /$ Water 1:1) were transferred into $200 \mu 1$ of $100 \mathrm{mM}$ phosphate buffer at $\mathrm{pH}$ 8. A solution of SPDP (starting from 5 eq of phallCn) in DMSO was added to the reaction mixture and stirred at room temperature. After about 2 hrs, most of the SPDP was hydrolyzed to PDP and no further progression of formation of the phallCn-PDP was observed. The $\mathrm{pH}$ of the reaction mixture was adjusted to pH 8 and more SPDP was added until almost all phallCn was reacted. The progress of the reaction was monitored using HPLC. Phallacidin- $\left(\mathrm{CH}_{2}\right)_{n}-\mathrm{SH}$ (phallCnSH) was obtained by reducing the disulfide bond in the phallCn-PDP using TCEP (20 eq to SPDP added) in 100 $\mathrm{mM}$ phosphate buffer $\mathrm{pH} 8$ for $30 \mathrm{~min}$, purified using reverse phase C18 HPLC, lyophilized, and characterized using ESI mass spectrometry. The elution times and MWs of phallotoxins on HPLC runs with 30 min gradients from 99:1 A/B to 70:30 A/B (flow rate $1 \mathrm{ml} / \mathrm{min}$ ) were: for phallacidin $30.3 \mathrm{~min} / 846.15 \mathrm{Da}$, for phallC 4 SH $32.4 \mathrm{~min} / 1005.17 \mathrm{Da}$, for

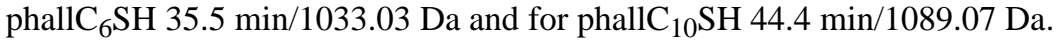

\section{Synthesis of pHLIP-S-S- $\left(\mathrm{CH}_{2}\right)_{n}$-phallacidin}

The lyophilized phall ${ }_{n}$-PDPs were dissolved in DMSO to have about $5 \mathrm{mM}$, followed by the addition of pHLIP peptide (2 eq) dissolved in DMSO and incubated at room temperature. More pHLIP was added if needed until almost all phallC $\mathrm{n}_{n}-\mathrm{PDPs}$ were reacted. 
The progress of the reaction was monitored using HPLC (flow rate $1 \mathrm{ml} / \mathrm{min}$; gradient 60 min from 99:1 A/B to 5:95 A/B). The $\mathrm{pHLIP}_{-} \mathrm{C}_{n}$ phall were analyzed using SELDI-TOF (surface-enhanced laser desorption/ionization time-of-flight) mass spectrometry and quantified by measuring OD at 280 and $300 \mathrm{~nm}$. $\left(\varepsilon_{280} / \varepsilon_{300}\right.$ of pHLIP and phallacidin is $13940 \mathrm{M}^{-1} \mathrm{~cm}^{-1} / 2999 \mathrm{M}^{-1} \mathrm{~cm}^{-1}$ and $10944 \mathrm{M}^{-1} \mathrm{~cm}^{-1} / 10100 \mathrm{M}^{-1} \mathrm{~cm}^{-1}$, respectively)

\section{Measurements of water-octanol partition coefficient}

The polarities of the phallotoxin cargoes were determined by assessment of relative partitioning between aqueous and octanol liquid phases. Constructs dissolved in MeOH:Water 1:1 were added to $0.5 \mathrm{ml}$ of $10 \mathrm{mM}$ phosphate buffer $\mathrm{pH} 5.5$ (saturated with argon) to concentrations of 20 and $30 \mu \mathrm{M}$, followed by the addition of argon saturated $n$ octanol $(0.5 \mathrm{ml})$ and sealed under argon. The solutions were mixed by rotation for $24 \mathrm{hrs}$ at room temperature and left for another 24-48 hrs to reach equilibrium. After phase separation, absorption at $300 \mathrm{~nm}$ was recorded. The molar extinction coefficients in $n$ octanol and phosphate buffer are assumed to be the same, and the ratio of the OD readings was used directly to calculate the partition coefficient, $P=\mathrm{OD}_{n \text {-octanol }} / \mathrm{OD}_{\text {water }}$, and $\log P$ values. A fraction of aqueous solution was analyzed using HPLC to ensure that no dimers of the phallotoxin were formed.

\section{Liposome preparations}

Liposomes were prepared by extrusion: POPC (1-palmitoyl-2-oleoyl-sn-glycero-3phosphocholine) ( $500 \mu \mathrm{l}$ of $10 \mathrm{mg} / \mathrm{ml}$ in chloroform) was transferred to a $100 \mathrm{ml}$ round bottom flask and a lipid layer was obtained by evaporating the choloroform in a rotary evaporator, followed by drying under high vacuum for $2 \mathrm{hrs}$. The lipid layer was resuspended in $10 \mathrm{mM}$ phosphate buffer, $\mathrm{pH} 8$, and extruded 31 times through a $50 \mathrm{~nm}$ membrane to obtain large unilamellar vesicles.

\section{Measurements of intrinsic peptide fluorescence and circular dichroism (CD) spectroscopic signals}

Intrinsic peptide fluorescence and circular dichroism (CD) spectra were measured on a PC1 ISS spectrofluorometer (ISS, Inc.) and a MOS-450 spectrometer (Bioligic, Inc.), respectively, under temperature control. All measurements were performed at $25^{\circ} \mathrm{C}$. Samples of $7 \mu \mathrm{M}$ of pHLIP- $\mathrm{C}_{n}$ phall incubated with $1.5 \mathrm{mM}$ POPC or in buffer $\mathrm{pH} 8$ overnight were used for the measurements of $\mathrm{CD}$ and fluorescence signals of the three states. $4 \mu \mathrm{M}$ of the pHLIP- $\mathrm{C}_{n}$ phall in $\mathrm{pH} 8$ incubated overnight were used for the experiment for aggregation of the peptide-cargo in aqueous medium. Peptide fluorescence spectra were recorded from $310 \mathrm{~nm}$ to $400 \mathrm{~nm}$ using excitation wavelengths of $280 \mathrm{~nm}$. Peptide CD spectra were recorded from $190 \mathrm{~nm}$ to $260 \mathrm{~nm}$ at $0.5 \mathrm{~nm}$ increments using a sample cuvette with an optical path length of $0.5 \mathrm{~cm}$.

\section{Binding assay}

Materials and preparation of stock solutions-Rabbit muscle actin was purchased from Cytoskeleton Inc. To obtain polymerized filamentous actin (F-actin), the monomeric globular actin (G-actin) was dissolved in $100 \mu \mathrm{l}$ of water and incubated for $1 \mathrm{hr}$ at room temperature. After centrifuging at 13,000×g for about $15 \mathrm{~min}$, the amount of G-actin in the supernatant was quantified by measuring the OD at $290 \mathrm{~nm}\left(\varepsilon_{290}\right.$ of G-actin is 26600 $\mathrm{M}^{-1} \mathrm{~cm}^{-1}$ ). G-actin was diluted to $3.5 \mathrm{mg} / \mathrm{ml}$ in $2 \mathrm{mM}$ phosphate buffer $\mathrm{pH} 8$ supplemented with $0.2 \mathrm{mM} \mathrm{CaCl}_{2}$ and $0.2 \mathrm{mM} \mathrm{ATP}$ and incubated for $1 \mathrm{hr}$ at $4{ }^{\circ} \mathrm{C}$. Polymerization was induced by addition of $50 \mathrm{mM} \mathrm{KCl}, 2 \mathrm{mM} \mathrm{MgCl}$ and $1 \mathrm{mM}$ ATP and incubation for $1 \mathrm{hr}$ at room temperature. Texas Red-X phalloidin (PhallTxR) was purchased from Invitrogen Corp. PhallTxR was dissolved in DMF and quantified by measuring OD at $583 \mathrm{~nm}$. $\left(\varepsilon_{583}\right.$ of 
PhallTx in $\mathrm{MeOH}$ is $95,000 \mathrm{M}^{-1} \mathrm{~cm}^{-1}$ ). Previously prepared and lyophilized phallotoxins (phallacidin, phallC4SH, phallC6SH and phallC10SH) were dissolved in DMSO and quantified by measuring OD at $300 \mathrm{~nm}$. ( $\varepsilon_{300}$ of phallotoxins $\left.10,100 \mathrm{M}^{-1} \mathrm{~cm}^{-1}\right)$.

Binding assay of phallTxR to actin-Samples of $0.6 \mu \mathrm{M}$ of phallTxR with different Factin concentrations (from 0 to $6.6 \mu \mathrm{M}$ ) were prepared in polymerizing buffer ( $2 \mathrm{mM}$ phosphate buffer pH8 containing $50 \mathrm{mM} \mathrm{KCl}, 2 \mathrm{mM} \mathrm{MgCl}_{2}, 0.2 \mathrm{mM} \mathrm{CaCl}_{2}$ and $1 \mathrm{mM}$ ATP) and incubated for $2 \mathrm{hrs}$ at room temperature. The fluorescence anisotropy and intensity of each sample were measured with excitation/emission setting at $570 \mathrm{~nm} / 610 \mathrm{~nm}$ wavelength, respectively under temperature control.

Competition binding assay-The assay is based on titration of $0.3 \mu \mathrm{M}$ of phallTxR and $0.3 \mu \mathrm{M}$ of phallotoxin by increasing concentrations of F-actin. 10× TCEP was added to 60 $\mu \mathrm{M}$ stock solution of phallotoxins in polymerizing buffer and incubated for $10 \mathrm{~min}$ to reduce disulfide bond. Samples of $0.3 \mu \mathrm{M}$ of phallTxR and $0.3 \mu \mathrm{M}$ of each phallotoxin were prepared in polymerizing buffer followed by mixing with F-actin to obtain final concentrations of $0,0.3,0.6,1.2$ and $2.4 \mu \mathrm{M}$ of F-actin in each sample, and incubated overnight at $4{ }^{\circ} \mathrm{C}$. The fluorescence anisotropy of each sample was measured with excitation/ emission setting to $570 \mathrm{~nm} / 610 \mathrm{~nm}$ measured using PC1 spectrofluorometer under temperature control.

\section{Cell line}

Human cervical adenocarcinoma HeLa was purchased from the American Tissue and Culture Collection (ATCC). HeLa was propagated in DMEM (Dulbecco's Modified Eagle Medium) ([+] $4.5 \mathrm{~g} / \mathrm{L}$ D-glucose, [+] $40 \mathrm{mg} / \mathrm{L}$ sodium pyruvate, Gibco) supplemented with $10 \%$ FBS (fetal bovine serum) (Gibco), ciprofloxacin- $\mathrm{HCl}(1 \mu \mathrm{g} / \mathrm{mL}$ ) (from Cellgro, Voigt Global Distribution) in a humidified atmosphere of $5 \% \mathrm{CO}_{2}$ at $37^{\circ} \mathrm{C}$. HeLa cells were adapted to $\mathrm{pH} 6.2$ by propagation in $\mathrm{pH} 6.2$ DMEM supplemented with $10 \%$ FBS, ciprofloxacin- $\mathrm{HCl}(1 \mu \mathrm{g} / \mathrm{mL})$ in humidified atmosphere of $5 \% \mathrm{CO}_{2}$ at $37^{\circ} \mathrm{C}$.

\section{Proliferation Assay}

Stock solutions of phallacidin, phalloidin-oleate, phall $_{n} \mathrm{SH}$ and $\mathrm{pHLIP}-\mathrm{C}_{n}$ phall were prepared in DMSO at $400 \mu \mathrm{M}$. A human cervix adenocarcinoma cell line (HeLa) obtained from the ATCC (American Type Culture Collection) was grown at pH 6.2 and 7.4. HeLa cells were seeded in 96-well plates (Costar) at densities of 4000 and 2000 cells per well for treatment on the following day. A DMSO stock of constructs were diluted with sterile Leibovitz's L-15 phenol free medium (L-15) pH 6.0 or 7.4 to give treatment solutions in the 0-6 $\mu \mathrm{M}$ range. Appropriate amounts of DMSO were added to ensure that all treatment samples contain the same amount of DMSO by volume (1.5\%). After removal of cell media, the $\mathrm{L}-15$ treatment solutions $\mathrm{pH} 6.0$ or $7.4(95 \mu \mathrm{L})$ were added to cells grown at $\mathrm{pH} 6.2$ and 7.4, respectively, and then the plate was incubated at $37^{\circ} \mathrm{C}$ for $3 \mathrm{hrs}$. After treatment, $200 \mu \mathrm{L}$ of DMEM at pH 6.2 or 7.4 were added to corresponding wells and $10 \mu 1$ of FBS into each well to provide $10 \%$ of FBS in cell medium before returning the plate to the incubator. Cell density of the ' $0 \mu \mathrm{M}, \mathrm{pH} 6.2$ ' and ' $0 \mu \mathrm{M}, \mathrm{pH} 7.4$ ' controls usually reached $80 \%-90 \%$ saturation in well after 4-6 days of growth. The viable cell number was quantified using the MTS reagent (Promega CellTiter 96 AQueous One Solution Cell Proliferation Assay). OD values at $490 \mathrm{~nm}$ were obtained using a plate reader (iMark Microplate reader from Bio $\mathrm{Rad})$. Since the rate of cells growth is slightly different at low and neutral $\mathrm{pHs}$, all numbers were normalized to $100 \%$ using wells where no construct was added to the media at various pHs. 


\section{Results}

\section{Design and synthesis of phallacidin cargoes of different hydrophobicity}

The major goal of our work is to systematically vary the hydrophobicity of a polar cargo, phallatoxin, and to investigate pHLIP-mediated $\mathrm{pH}$-dependent cellular delivery of constructs of different polarity of cargoes. We chose phallacidin (Figure 1), which is a cyclic cellimpermeable toxin similar to phalloidin and that binds to F-actin with high affinity $(18,19)$. Phallacidin has a free $\mathrm{COOH}$ group suitable for conjugation purposes, and we tuned the hydrophobicity of the phallacidin cargo by reacting with diamines $\mathrm{NH}_{2}-\left(\mathrm{CH}_{2}\right)_{n}-\mathrm{NH}_{2}$ with different lengths of hydrophobic chain $\left(\mathrm{CH}_{2}\right)_{n}$, where $n$ could vary from 2 to 12 carbon atoms. We had already tested commercially available phalloidin-oleate, with 15 carbon atoms, which has $\log P$ value of +1.7 , and observed inhibition of Hela cell proliferation rate by $60 \%, 70 \%$ and $95 \%$ after treatment cells with 1,2 and $4 \mu \mathrm{M}$ of phalloidin-oleate, respectively (data not shown), which indicate that attachment of 15 carbon atoms to phalloidin makes it too hydrophobic, so it can easily cross lipid bilayer itself. We selected 3 different lengths of hydrophobic chain diamines, where $n$ is 4,6 and 10 to work with. The phallacidin was conjugated to the various lengths diamines via NHS and DCC crosslinker. We synthesized three phallacidin cargoes with four (phallC4), six (phallC6) and ten (phallC10) carbon atoms. The protocol was adjusted until optimal conditions were established, and the details of the final protocol are in the Methods section. The products were purified using reverse phase C18 HPLC, lyophilized and characterized by ESI mass spectrometry. The molecular weights obtained for phallacidin cargoes were 917.2 Da for phallC4, 945.2 Da for phallC6 and 1001.2 Da for phallC10 and were very close to expected values $(917.1,945.1$ and $1001.2 \mathrm{Da})$.

\section{Characterization of phallacidin cargoes of different hydrophobicity}

To investigate the properties of cargo molecules, they were reacted with SPDP crosslinker, the S-S bond was reduced by TCEP and the reduced cargoes were purified and characterized (see Table 1). The cargo hydrophobicities were evaluated by measuring the logarithm of the octanol-water partition coefficient $P$, calculated based on the amount of constructs distributed upon equilibration between octanol and water phases, measured by the ODs of phallacidin constructs at $300 \mathrm{~nm}$. The $\log P$ values of phallacidin and cargoes are presented in Table 1. Phallacidin with a long chain FA of ten carbon atoms is preferably distributed into octanol, being hydrophobic, and shows a positive $\log P$ of +1.28 . Such molecules are expected to cross cellular membranes by themselves (at least at high concentrations), the hydrophobicity being in the range of conventional drugs. The polarity of phallC6SH with $\log P=-0.09$ was very close to the polarity of phalloidin-rhodamine, which has $\log P=$ -0.05 measured previously (20). Phallacidin with four carbon atoms, as expected, was the most polar among modified phallacidin cargoes.

According to the literature $(18,21,22)$ it was expected that modification of $\mathrm{COOH}$ group of phallacidin should not affect F-actin binding properties. Phallatoxin binds between actin monomers in filamentous actin and prevents depolymerization $(19,21)$. We used a fluorescence anisotropy titration assay, where phalloidin conjugated to Texas Red (TxR) fluorescent dye was in competition with phallacidin and cargoes for F-actin binding. The assay is based on the increase of anisotropy of phallTxR when it binds to the F-actin. Samples of equal concentration $(0.3 \mu \mathrm{M})$ of phallTxR and phallacidin cargoes were prepared with increasing concentrations of F-actin. The samples were incubated overnight, then the fluorescence anisotropy of each sample was measured at $610 \mathrm{~nm}$ wavelength with excitation at $570 \mathrm{~nm}$ (Figure 2). The anisotropy changes from 0.04 (for unbound phallTxR) up to 0.24 when all phallTxR is completely bound (the value of 0.24 was obtained in separate titration experiment of phalloidinTxR by F-actin in the absence of phallacidin or 
phallacidin cargoes). Our experiments demonstrate that the anisotropy value in the presence of phallacidin cargoes changes in the same way as anisotropy in the presence of phallacidin, confirming that the attachment of hydrophobic tails to the phallacidin does not alter binding affinity to F-actin.

\section{Synthesis and characterization of pHLIP- $\mathrm{C}_{n}$ phall}

We synthesized pHLIP-C4phall, pHLIP-C6phall and pHLIP-C10phall constructs, purified them, performed spectroscopic characterization and tested their anitproliferative properties. $\mathrm{PhallC}_{\mathrm{n}}$-PDP were conjugated with single Cys residue at the C-terminus of pHLIP to form a $\mathrm{S}-\mathrm{S}$ bond. The products were purified using reverse phase C18 HPLC, lyophilized and characterized by SELDI-TOF mass spectrometry (MW of the pHLIP-C4phall, pHLIPC6phall and pHLIP-C10phall were 5120.1, 5155.4 and 5204.2 Da respectively) and quantified by measuring OD at 280 and $300 \mathrm{~nm}$.

We have demonstrated previously that changes of intrinsic fluorescence and CD of pHLIP in the presence of liposomes resulting from $\mathrm{pH}$ changes is indicative of peptide insertion into the lipid bilayer $(4,5)$. Here, we carried out spectroscopic characterization of pHLIP- ${ }_{n}$ phall constructs (Figure 3). We found that all constructs are predominantly unstructured in aqueous solution at $\mathrm{pH} 8$, while the position of the maximum of fluorescence is slightly shifted to short wavelengths when cargo with longer carbon chain was conjugated to pHLIP (Table 2). The shift of the position of maximum is accompanied with the increase of ellipticity at $222 \mathrm{~nm}$. All spectroscopic data indicate that pHLIP-C10phall most probably is more aggregated compared to pHLIP, pHLIP-C4phall and pHLIP-C6phall. More pronounced differences between constructs were observed at $\mathrm{pH} 8$ in the presence of POPC. The highest amount of helical structure and the deepest partition of the emitting residues into the core of bilayer were observed for the pHLIP-C10phall. The lowering of $\mathrm{pH}$ from 8 to 4 induced further increase of helical content and partition of the constructs into the membrane accompanied with the increase of fluorescence and shift of the emission to the short wavelengths. The $\mathrm{pH}$-induced fluorescence and $\mathrm{CD}$ changes seen for different constructs in the presence of lipid bilayer were very similar to those observed for pHLIP alone. These changed were more significant for pHLIP-C10phall compared to pHLIP$\mathrm{C} 4$ phall. Thus, we concluded that conjugation of phallC $\mathrm{C}_{n}$ cargoes, in general, does not affect the $\mathrm{pH}$-dependent ability of pHLIP to interact with the membrane lipid bilayer.

\section{Antiproliferative effect of $\mathrm{pHLIP}-\mathrm{C}_{\mathrm{n}}$ phall}

Finally, we tested the anitproliferative capability of the pHLIP- $\mathrm{C}_{n}$ phall constructs. $\mathrm{HeLa}$ cells were adapted for low $\mathrm{pH}$ growth ( $\mathrm{pH}$ 6.2). Cells grown at low and normal (7.4) $\mathrm{pHs}$ were treated with increasing concentrations of the $\mathrm{pHLIP}-\mathrm{C}_{\mathrm{n}}$ phall, phallC $\mathrm{C}_{n}$ and phallacidin in L-15 phenol free medium at $\mathrm{pH} 6.0$ and 7.4 for $3 \mathrm{hrs}$. After treatment DMEM supplemented with $10 \%$ FBS at pH 6.2 or 7.4 was added to corresponding cells. When cell density in the control wells (treated with medium) reached 80\%-90\% saturation (after 4-6 days of growth) the number of viable cells was quantified using the MTS reagent. Since the rate of cells growth is slightly different at low and neutral pHs, all numbers were normalized to be $100 \%$ where no construct was added to the media. Phallacidin and phallC $\mathrm{C}_{n}$ cargoes do not demonstrate an antiproliferative effect at either $\mathrm{pHs}$ (data not shown). At the same time, the data clearly demonstrate that pHLIP-C4phall and pHLIP-C6phall show concentration dependent antiproliferative effect only at low $\mathrm{pH}$ of treatment, while at neutral $\mathrm{pH}$ no effect was observed (Figure 4). At $2 \mu \mathrm{M}$ of pHLIP-C4phall and pHLIP-C6phall about 30\% and $70 \%$, respectively, of cell death was observed at low $\mathrm{pH}$. As we expected, the increase of cargo hydrophobicity correlates with the enhancement of antiproliferative effect. However, surprisingly, we did not observe expected biological effect, when cells were treated with pHLIP-C10phall. 


\section{Conformational states of pHLIP-C10phall in solution at different pHs}

Since we treated cells with constructs at pH5.9-6.0 for 3 hours, the constructs were dissolved in media at low $\mathrm{pH}$. Previously, we demonstrated that pHLIP has tendency for the aggregation in solution, when $\mathrm{pH}$ is lowered from 8-7.4 to 4-3 (5). When various cargoes are attached to the peptide the overall hydrophobicity is altered, and solubility could be changed as well. The overall hydrophobicity of the pHLIP-C10phall is higher than the hydrophobicity of the pHLIP-C4phall, therefore, the tendency for the aggregation is higher for the pHLIP-C10phall. We compared fluorescence and CD signals of pHLIP-C10phall in

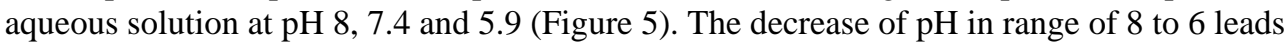
to the conformational changes accompanied by the shift of the position of maximum of fluorescence from 349 to $346 \mathrm{~nm}$, the increase of amount of helical structure and the reduction of quantum yield of fluorescence. These spectral changes were not observed in case of pHLIP-C4phall and pHLIP-C6phall in the range of pH8-6 (data not shown). We concluded that pHLIP-C10phall in solution at pH6 is partially aggregated, which alters its interaction with the lipid bilayer of cellular membrane and leads to the reduction of anitproliferative effect.

\section{Discussion}

In conventional drug design and discovery the Lipinski rules of five, or subsequently developed similar parameters, are widely used to guide drug designs. The rules postulate that a successful drug should be hydrophobic and small in order to traverse membranes and reach cytoplasmic targets (e.g. the logarithm of the octanol-water partition coefficient $\log P$ is -0.4 to +5.6 and the MW is 160 to $480 \mathrm{~g}^{\mathrm{mol}}{ }^{-1}$ ) (23). However, the majority of inhibitors found for biological targets located inside a cell are molecules that cannot cross a membrane (24-26). We have proposed a novel way to deliver polar molecules across membranes, based on the insertion of a water-soluble, moderately hydrophobic membrane peptide, pHLIP. The spontaneous insertion and folding of the peptide into a lipid bilayer seeks the free energy minimum, and the insertion event is therefore accompanied by a release of energy, which is used to translocate cell-impermeable cargo molecules across a cellular membrane. The Gibbs Free Energy of binding of pHLIP to a POPC surface at $37^{\circ} \mathrm{C}$ is about $-7 \mathrm{kcal} / \mathrm{mol}$ at $\mathrm{pH} 8$ and the additional free energy of insertion and folding across a lipid bilayer induced by reduction of $\mathrm{pH}$ from 8 to 4 is nearly $-2 \mathrm{kcal} / \mathrm{mol}$ (13). The energy difference between membrane-bound and membrane-inserted states favors the partition of cargo across the hydrophobic barrier of a membrane. Since the energy released during peptide folding and insertion across a membrane is limited, and since strongly polar molecules will reach equilibrium slowly, we assumed that there is a limit on cargo polarity (and most probably on size as well) that can be delivered across a membrane by pHLIP. In support of that, we recently show that pHLIP can move phalloidin across a membrane to inhibit cell proliferation, but only when a hydrophobic facilitator (rhodamine) is attached to the peptide inserting end. In this study we made a first attempt to tune hydrophobicity of polar cargo phallacidin in a systematic manner by conjugation of phallacidin with diamines of different hydrophobic chain lengths. The hydrophobicity of the cargo is modulated by presence of 4 to 10 carbon atoms conjugated to the carboxyl group of phallacidin. The cargoes were synthesized and characterized. We show that the logarithm of the octanol-water partition coefficient $(\log P)$ of cargoes was varied from -1.6 for pure phallacidin to +1.28 for phallC10. Cargo to be functional needs to bind to its cellular target, in the case of phallacidin it is an F-actin. We evaluated the actin-binding ability of cargoes and demonstrated that attachment of a chain of carbon atoms (up to 10 atoms) to the carboxyl group of phallacidin does not affect their ability to interact with F-actin. Next, the phallC $\mathrm{C}_{\mathrm{n}}$ cargoes were conjugated via a cleavable $\mathrm{S}-\mathrm{S}$ bond to the $\mathrm{C}$-terminus of $\mathrm{pHLIP}$, which goes across the membrane. First, we carried out spectroscopic characterization of pHLIP- ${ }_{n}$ phall 
cargoes. The experiments were done on model system of POPC liposomes. The constructs, first, were dissolved in aqueous solution at $\mathrm{pH} 8$, then pre-incubated with liposomes at $\mathrm{pH} 8$, and, after that, the $\mathrm{pH}$ was dropped from 8 to 4 . The conditions of model experiment differ from conditions of the experiments on cultured cells, while the conditions were chosen to ensure completion of all transitions and for the comparison to our previously published biophysical studies (4-13). In general, we observed characteristic changes of the fluorescence and CD signals, when pHLIP-C $\mathrm{C}_{n}$ phall interact with lipid bilayer of a POPC membrane at neutral and low $\mathrm{pHs}$ as it was observed for pHLIP peptide (4-13). The changes of spectroscopic signals were the most pronounced for the pHLIP-C10phall compared to the pHLIP-C4phall. It was an important sign, indicating that attachment of phall $\mathrm{C}_{\mathrm{n}}$ cargo molecules to the inserting end of the peptide does not prevent $\mathrm{pH}$-dependent membrane interaction of the peptide. Next, we carried out cell experiments to demonstrate that phall $\mathrm{C}_{\mathrm{n}}$ could be translocated across a membrane by pHLIP to induce inhibition of cell proliferation. It is known that phallacidin in the cytoplasm stabilizes actin in its filamentous form, preventing depolymerisation. Since cell movement and shape changes are associated with actin polymerization/depolymerisation, stabilization of F-actin depolymerisation leads to the freezing of cell movement, formation of multinucleated cells and eventually cell death (8, 20). Phallacidin and phall $\mathrm{C}_{\mathrm{n}}$ did not show any biological effect at both $\mathrm{pHs}$, while pHLIP facilitated translocation of phallC4 and phallC6 cargoes at slightly acidic $\mathrm{pH}$, which in turns led to the inhibition of cell proliferation in a concentration-dependent manner. As we expected, the antiproliferative effect was more significant in case of cell treatment with pHLIP-C6phall compared to pHLIP-C4phall, since phallC6 is more hydrophobic than phallC4. However, surprisingly we did not monitor the desired biological effect when cells were treated with pHLIP-C10phall. Additional characterization of the conformation states of pHLIP-C10phall in aqueous solutions in range of pH8-6 revealed that the construct has much higher tendency for aggregation at $\mathrm{pH} 6$ (pH used to treat cells) in comparison to pHLIP, pHLIP-C4phall and pHLIP-C6phall. As a result, the efficacy of treatment was reduced. We concluded that increase of cargo hydrophobicity indeed facilitates its translocation across membrane by pHLIP, however the requirement of construct solubility is critical, since construct interaction will cellular membrane could be altered leading to the reduced translocation ability and diminished biological effect.

In contrast to all other known peptide-based delivery technologies, selective delivery of molecules into the cytoplasm by pHLIP is achieved by the $\mathrm{pH}$-dependent folding of a monomeric peptide across the plasma membrane. In response to the low extracellular $\mathrm{pHs}$ of cells in diseased tissues, pHLIP can translocate polar therapeutic cargo molecules into cell cytoplasms, whereas at the normal extracellular $\mathrm{pH}$ of healthy tissue, only a minimal translocation of cargo across cell membranes would occur. Because the cargo is translocated across a cell membrane directly into the cytoplasm, endosomal trapping is avoided. Tuning the cargo hydrophobicity can be used to achieve the maximum difference between the therapeutic effect at low $\mathrm{pH}$ versus at neutral $\mathrm{pH}$, thereby enhancing diseased-targeted delivery and reducing treatment side effects. However, to achieve the desired enhancement of therapeutic efficacy, it is critical to ensure construct solubility, which could be reduced due to the increase of cargo hydrophobicity.

\section{Acknowledgments}

We thank Dr. Anna Moshnikova and Erin Jansen (Physics, URI) for help with cells experiments and purification of constructs, respectively; Prof. Brenton DeBoef and Dr. Shathaverdhan Potavathri (Chemistry, URI) for guidance in phallcidin cargo synthesis; Dr. Ming An (Molecular Biophysics and Biochemistry, Yale), Dr. Gregory Watkins (Delpor, Inc.) for discussion.

This work was supported by NIH grant CA133890 to OAA, DME, YRK, and by DoD grant PC050351 to YKR. 


\section{References}

1. Jeffrey SS, Lonning PE, Hillner BE. Genomics-based prognosis and therapeutic prediction in breast cancer. J Natl Compr Canc Netw. 2005; 3:291-300. [PubMed: 16002001]

2. Krebs HA. The Pasteur effect and the relations between respiration and fermentation. Essays Biochem. 1972; 8:1-34. [PubMed: 4265190]

3. Warburg O, Wind F, Negelein E. The metabolism of tumors in the body. J Gen Physiol. 1927; 8:519-530. [PubMed: 19872213]

4. Hunt JF, Rath P, Rothschild KJ, Engelman DM. Spontaneous, pH-dependent membrane insertion of a transbilayer alpha-helix. Biochemistry. 1997; 36:15177-15192. [PubMed: 9398245]

5. Reshetnyak YK, Segala M, Andreev OA, Engelman DM. A monomeric membrane peptide that lives in three worlds: in solution, attached to, and inserted across lipid bilayers. Biophys J. 2007; 93:2363-2372. [PubMed: 17557792]

6. Andreev OA, Engelman DM, Reshetnyak YK. Targeting acidic diseased tissue: New technology based on use of the pH (Low) Insertion Peptide (pHLIP). Chemistry Today. 2009; 27:34-37. [PubMed: 20037661]

7. Andreev OA, Engelman DM, Reshetnyak YK. pH-sensitive membrane peptides (pHLIPs) as a novel class of delivery agents. Mol Membr Biol. 2010; 27:341-352. [PubMed: 20939768]

8. Reshetnyak YK, Andreev OA, Lehnert U, Engelman DM. Translocation of molecules into cells by pH-dependent insertion of a transmembrane helix. Proc Natl Acad Sci U S A. 2006; 103:64606465. [PubMed: 16608910]

9. Thevenin D, An M, Engelman DM. pHLIP-mediated translocation of membrane-impermeable molecules into cells. Chem Biol. 2009; 16:754-762. [PubMed: 19635412]

10. Andreev OA, Dupuy AD, Segala M, Sandugu S, Serra DA, Chichester CO, Engelman DM, Reshetnyak YK. Mechanism and uses of a membrane peptide that targets tumors and other acidic tissues in vivo. Proc Natl Acad Sci U S A. 2007; 104:7893-7898. [PubMed: 17483464]

11. Musial-Siwek M, Karabadzhak A, Andreev OA, Reshetnyak YK, Engelman DM. Tuning the insertion properties of pHLIP. Biochim Biophys Acta. 2010; 1798:1041-1046. [PubMed: 19766589]

12. Barrera FN, Weerakkody D, Anderson M, Andreev OA, Reshetnyak YK, Engelman DM. Roles of Carboxyl Groups in the Transmembrane Insertion of Peptides. J Mol Biol. 2011; 413:359-371. [PubMed: 21888917]

13. Reshetnyak YK, Andreev OA, Segala M, Markin VS, Engelman DM. Energetics of peptide (pHLIP) binding to and folding across a lipid bilayer membrane. Proc Natl Acad Sci U S A. 2008; 105:15340-15345. [PubMed: 18829441]

14. Andreev OA, Karabadzhak AG, Weerakkody D, Andreev GO, Engelman DM, Reshetnyak YK. pH (low) insertion peptide (pHLIP) inserts across a lipid bilayer as a helix and exits by a different path. Proc Natl Acad Sci U S A. 2010; 107:4081-4086. [PubMed: 20160113]

15. Tang J, Gai F. Dissecting the membrane binding and insertion kinetics of a pHLIP peptide. Biochemistry. 2008; 47:8250-8252. [PubMed: 18636715]

16. Vavere AL, Biddlecombe GB, Spees WM, Garbow JR, Wijesinghe D, Andreev OA, Engelman DM, Reshetnyak YK, Lewis JS. A novel technology for the imaging of acidic prostate tumors by positron emission tomography. Cancer Res. 2009; 69:4510-4516. [PubMed: 19417132]

17. Reshetnyak YK, Yao L, Zheng S, Kuznetsov S, Engelman DM, Andreev OA. Measuring Tumor Aggressiveness and Targeting Metastatic Lesions with Fluorescent pHLIP. Mol Imaging Biol. 2010

18. Wieland T. Modification of actins by phallotoxins. Naturwissenschaften. 1977; 64:303-309. [PubMed: 18683]

19. Barak LS, Yocum RR. 7-Nitrobenz-2-oxa-1,3-diazole (NBD) - phallacidin: synthesis of a fluorescent actin probe. Analytical Biochemistry. 1981; 110:31-38. [PubMed: 6894219]

20. An M, Wijesinghe D, Andreev OA, Reshetnyak YK, Engelman DM. pH-(low)-insertion-peptide (pHLIP) translocation of membrane impermeable phalloidin toxin inhibits cancer cell proliferation. Proc Natl Acad Sci U S A. 2010; 107:20246-20250. [PubMed: 21048084] 
21. Wieland T, Hollosi M, Nassal M. Components of the green deathcap mushroom (Amanita phalloides), LXI: delta-Aminophalloin, a 7-analogue of phalloidin, and some biochemically useful, including fluorescent derivatives. Liebigs Ann Chem. 1983; 1983:1533-1540.

22. Falcigno L, Costantini S, D'Auria G, Bruno BM, Zobeley S, Zanotti G, Paolillo L. Phalloidin synthetic analogues: structural requirements in the interation with F-actin. Chem Eur J. 2001; 7:4665-4673. [PubMed: 11757659]

23. Lipinski CA, Lombardo F, Dominy BW, Feeney PJ. Experimental and computational approaches to estimate solubility and permeability in drug discovery and development settings. Adv Drug Deliv Rev. 2001; 46:3-26. [PubMed: 11259830]

24. Tsutsumi S, Neckers L. Extracellular heat shock protein 90: a role for a molecular chaperone in cell motility and cancer metastasis. Cancer Sci. 2007; 98:1536-1539. [PubMed: 17645779]

25. Wang JL, Zhang ZJ, Choksi S, Shan S, Lu Z, Croce CM, Alnemri ES, Korngold R, Huang Z. Cell permeable Bcl-2 binding peptides: a chemical approach to apoptosis induction in tumor cells. Cancer Res. 2000; 60:1498-1502. [PubMed: 10749111]

26. Sun H, Nikolovska-Coleska Z, Yang CY, Qian D, Lu J, Qiu S, Bai L, Peng Y, Cai Q, Wang S. Design of small-molecule peptidic and nonpeptidic Smac mimetics. Acc Chem Res. 2008; 41:1264-1277. [PubMed: 18937395] 


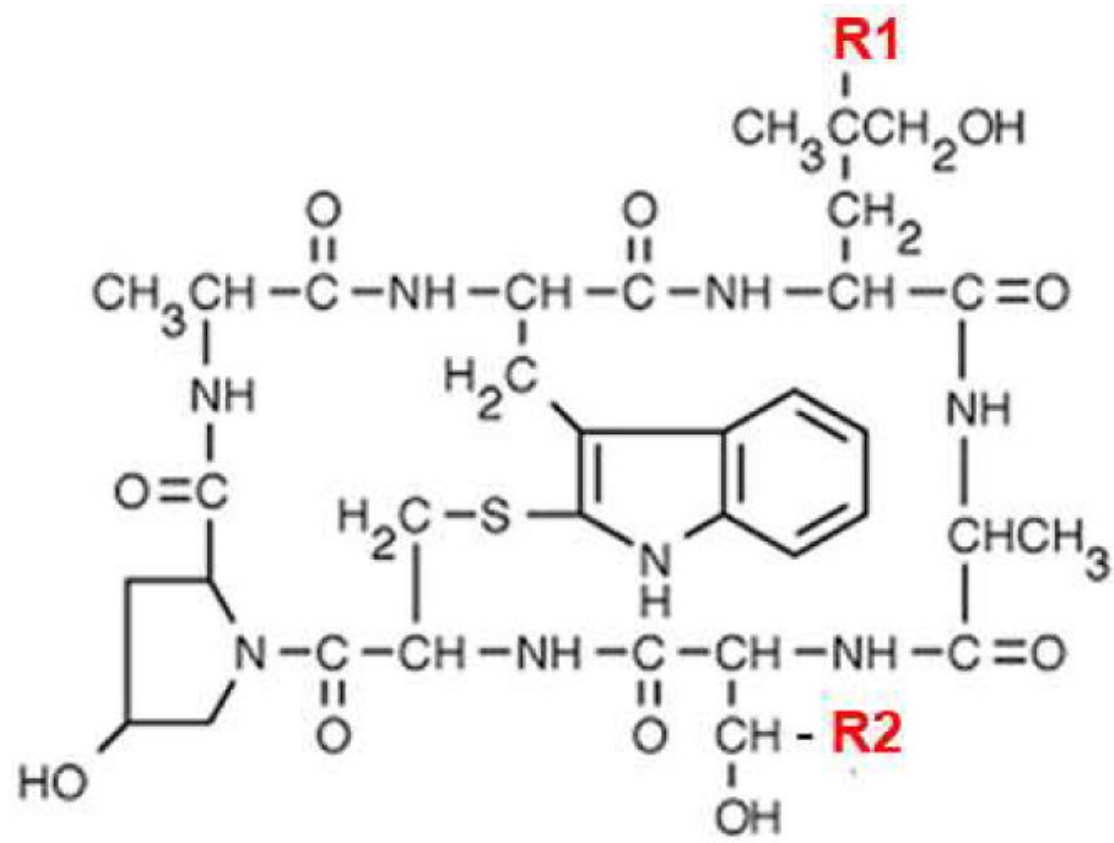

Figure 1.

Chemical structures of cyclic peptides: phalloidin, where $\mathrm{R} 1=\mathrm{OH}$ and $\mathrm{R} 2=\mathrm{CH}_{3}$; phallodin-rhodamine where $\mathrm{R} 1$ = rhodamine; and phallacidin, where $\mathrm{R} 1=\mathrm{H}$ and $\mathrm{R} 2=$ $\mathrm{COOH}$. Diamines consisting of different length of carbon chains $\left(\mathrm{CH}_{2}\right)_{4},\left(\mathrm{CH}_{2}\right)_{6}$ and $\left(\mathrm{CH}_{2}\right)_{10}$ were attached to the $\mathrm{COOH}$ group (R2). 


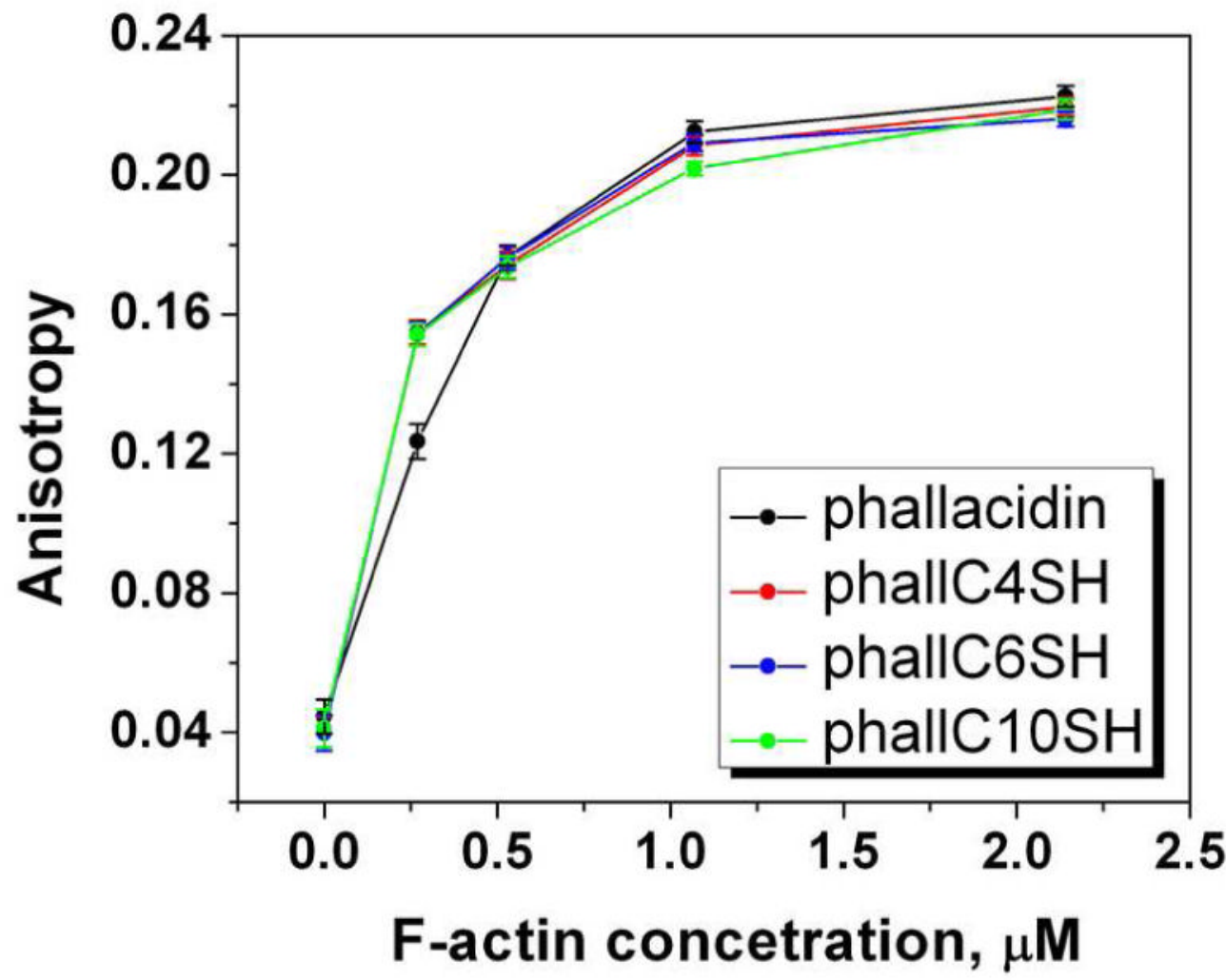

Figure 2.

Binding competition assay. Changes of fluorescence anisotropy of Texas Red conjugated to phalloidin $(0.3 \mu \mathrm{M})$ was monitored in the presence of phallacidin or phallacidin cargoes $(0.3$ $\mu \mathrm{M})$ at different F-actin concentrations. 

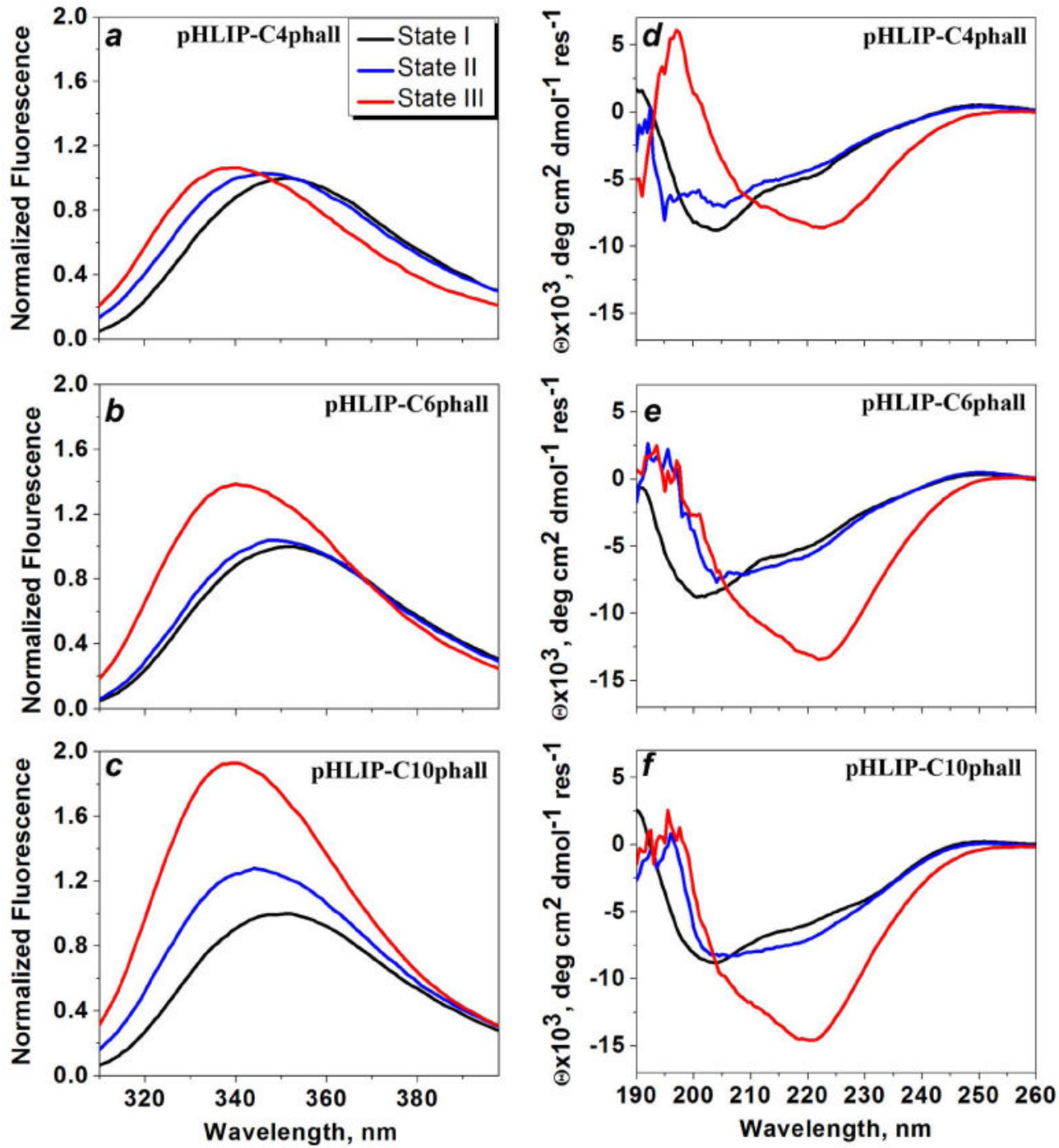

Figure 3.

Changes of intrinsic fluorescence $(\boldsymbol{a}-\boldsymbol{c})$ and CD $(\boldsymbol{d}-\boldsymbol{f})$ spectral signals upon pHLIP-C4phall, pHLIP-C6phall and pHLIP-C10phall interaction with POPC liposomes at various pHs. Black lines represent spectra for the construct in aqueous solution at $\mathrm{pH}$, blue lines upon incubation with POPC liposomes at $\mathrm{pH} 8$, and red lines after the $\mathrm{pH}$ was dropped from 8 to 4 . 


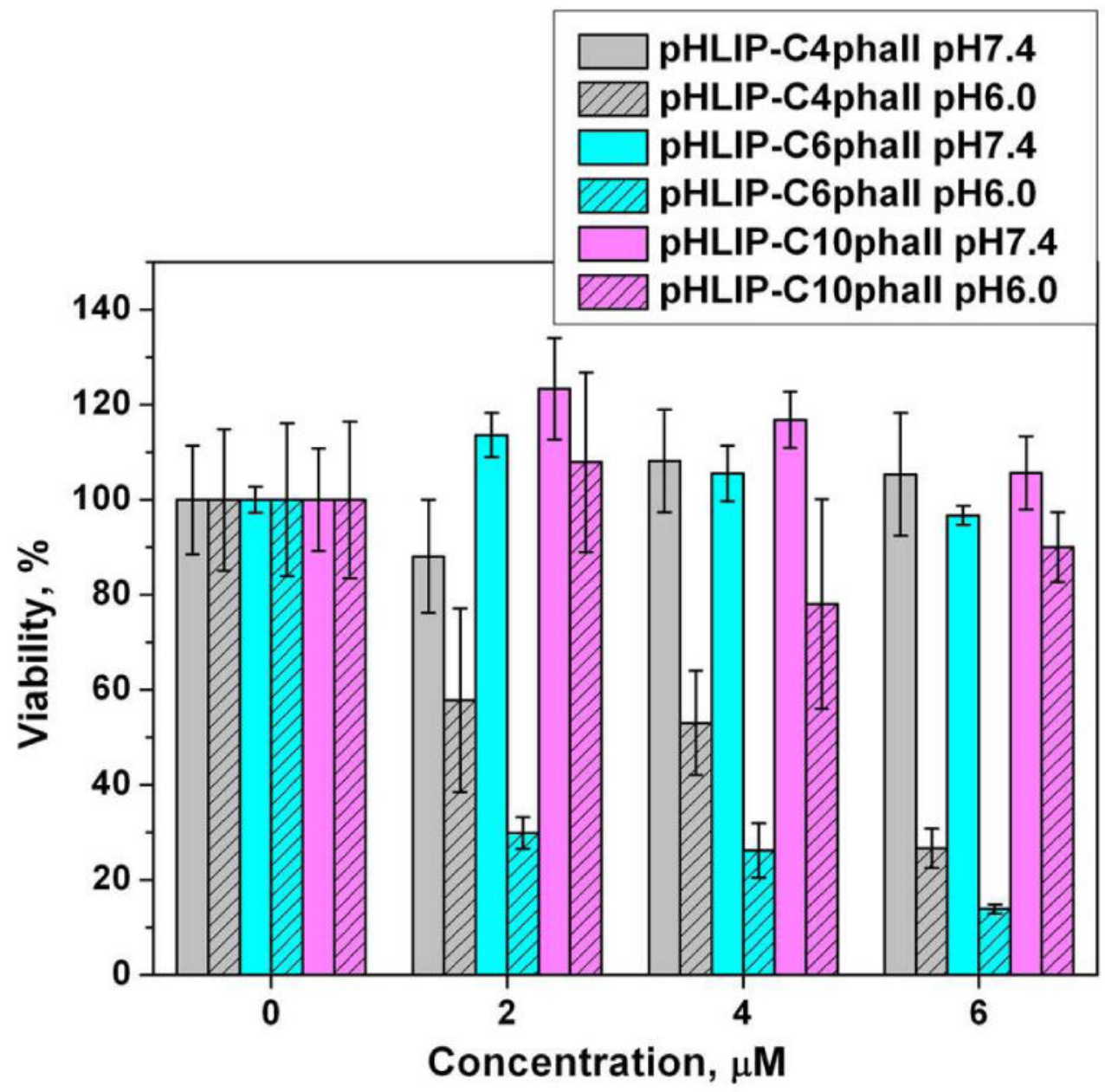

Figure 4.

Inhibition of cell proliferation by pHLIP-C4phall, pHLIP-C6phall, pHLIP-C10phall at low $\mathrm{pH}$. 

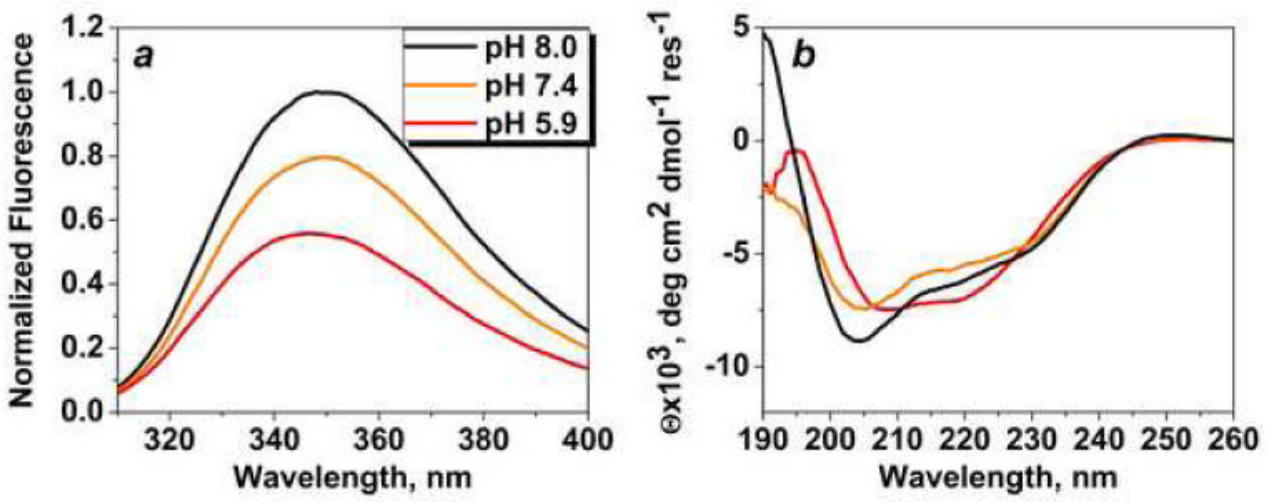

Figure 5.

Intrinsic peptide fluorescence $(\boldsymbol{a})$ and $\mathrm{CD}(\boldsymbol{b})$ spectral signals of pHLIP-C10phall at various pHs. 


\section{Table 1}

Characterization of phallacidin and phallalacidin-C4, -C6 and - $\mathrm{C} 10$ cargoes: percentage of acetonitrile of cargo elutions from the column and the logarithms of the octanol-water partition coefficients $(\log P)$.

\begin{tabular}{|l|c|c|}
\hline & Acetonitrile, \% & $\log \boldsymbol{P}$ \\
\hline phallacidin & 20.3 & -1.6 \\
\hline phallC4SH & 22.4 & -0.74 \\
\hline phallC6SH & 25.5 & -0.09 \\
\hline phallC10SH & 34.4 & +1.28 \\
\hline
\end{tabular}




\section{Table 2}

The spectral parameters of the pHLIP-C4phall, pHLIP-C6phall and pHLIP-C10phall in the states I, II and III are presented. The parameters were obtained in the result of the analysis of the fluorescence and CD spectra shown on the Figure 3: the maximum position of the fluorescence spectrum $\lambda_{\max }, S-$ the normalized area under the spectra (normalization was done on the area under the spectrum in the state I); $\theta_{222} \times 10^{3}, \mathrm{deg}^{2}$ $\mathrm{dmol}^{-1} \mathrm{res}^{-1}$ - the molar ellipticity at $222 \mathrm{~nm}$.

\begin{tabular}{|l|l|l|l|l|}
\hline & State I & State II & State III \\
\hline \multirow{4}{*}{ pHLIP-C4phall } & $\lambda_{\max }$ & $350.9 \mathrm{~nm}$ & $346.6 \mathrm{~nm}$ & $339.7 \mathrm{~nm}$ \\
& $\boldsymbol{S}$ & 1.0 & 1.08 & 1.05 \\
& $\boldsymbol{\theta}_{\mathbf{2 2 2}}$ & -4.6 & -4.1 & -6.05 \\
\hline \multirow{5}{*}{ pHLIP-C6phall } & $\lambda_{\max }$ & $349.9 \mathrm{~nm}$ & $348.3 \mathrm{~nm}$ & $340.2 \mathrm{~nm}$ \\
& $\boldsymbol{S}$ & 1.0 & 1.03 & 1.31 \\
& $\boldsymbol{\theta}_{\mathbf{2 2 2}}$ & -4.6 & -5.2 & -13.4 \\
\hline & $\lambda_{\max }$ & $349.6 \mathrm{~nm}$ & $344.6 \mathrm{~nm}$ & $339.2 \mathrm{~nm}$ \\
pHLIP-C10phall & $\boldsymbol{S}$ & 1.0 & 1.28 & 1.82 \\
& $\boldsymbol{\theta}_{\mathbf{2 2 2}}$ & -5.9 & -7.0 & -14.6 \\
\hline
\end{tabular}

